Chemistry and Technology of Fuels and Oils, Vol. 42, No.4, 2006

\title{
METHODS OF ANALYSIS
}

\section{SCANNING CALORIMETRY AND THERMOGRAVIMETRY IN ANALYSIS OF \\ PETROLEUM SYSTEMS. DETERMINATION OF THE COMPONENT COMPOSITION}

\author{
Yu. L. Shishkin
}

UDC 536.62:536.63

The possibility of qualitative analysis of heavy hydrocarbon mixtures by differential scanning calorimetry (DSC) and quantitative analysis combined with thermogravimetry (TG) was demonstrated. In experimental conditions that ensure complete oxidation of the sample on heating, successive oxidation and evaporation of paraffins, light and heavy aromatics, and naphthenes, resins, asphaltenes, and carbenes take place. Crudes of different classes (waxy, naphthene-paraffin, naphthene bases) and geological age were investigated. Methods of qualitative and quantitative analysis by TG-DSC are reported.

Determining the chemical (group, structural-group) composition of crude oils and their cuts is a pressing problem in refining, geological-exploration work, environmental monitoring, and control-analytical and arbitration operations. Standard methods are usually used for analysis, but they do not always satisfy the requirements for speed, simplicity, and convenience. For this reason, attempts to supplement or replace these methods with faster and more informative methods involving the latest advances in science and instrument-building technology for developing them have not stopped.

Of the existing instrumental methods of evaluating different properties and chemical composition, calorimetric and thermogravimetric methods should be mentioned first [1-2]. Heating the sample at a given rate (usually $10-50 \% \mathrm{~min}$ ) at different composition and pressure of the gas medium and recording a certain parameter as a function of the temperature, in the given case, the mass or amount of released/absorbed heat, are characteristic of both methods. Combining different methods with simultaneous measurement of several parameters of the object is especially informative.

Heating such a complex multicomponent system as crude oil results in evaporation of first light IBP-180 (200) ${ }^{\circ} \mathrm{C}$ naphtha-ligroin cuts, then 200-300 (320 ${ }^{\circ} \mathrm{C}$ kerosene-gasoil cuts, and finally, $>300^{\circ} \mathrm{C}$ lube oil

cuts. Atmospheric or vacuum distillation of petroleum systems used for qualitative and quantitative evaluation of

I. M. Gubkin Russian State University of Oil and Gas. Translated from Khimiya i Tekhnologiya Topliv $i$ Masel, No. 4, pp. 48 - 52, July-August, 2006. 


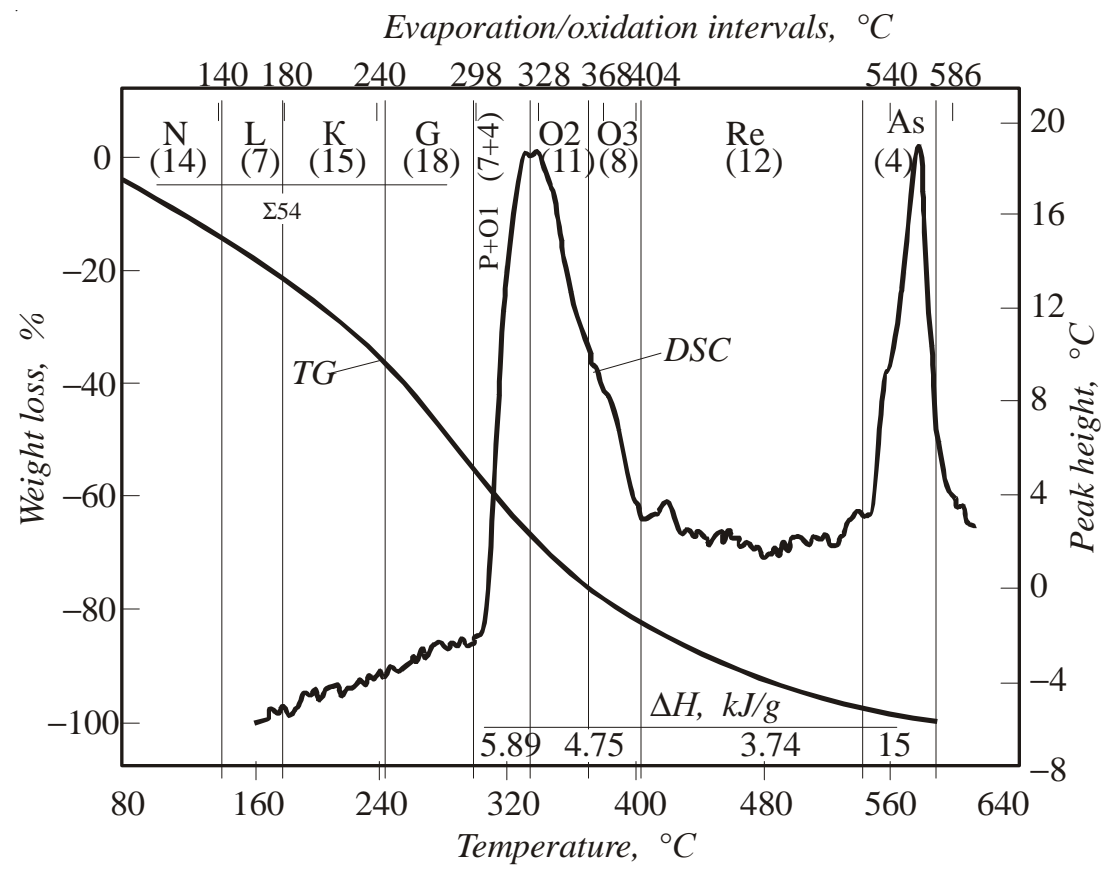

Fig. 1. TG-DSC curves of crude from Blinov field (Kazakhstan): $N, L, K, G$ - respectively naphtha, ligroin, kerosene, and gasoil cuts; $P+O 1$ - paraffins and light oil cuts; $O 2, O 3$ - middle and heavy oil cuts; $R e$ - resins; As - asphaltenes; in parentheses: content of components, $\% ; \Delta H$ - specific heat of oxidation of the corresponding components.

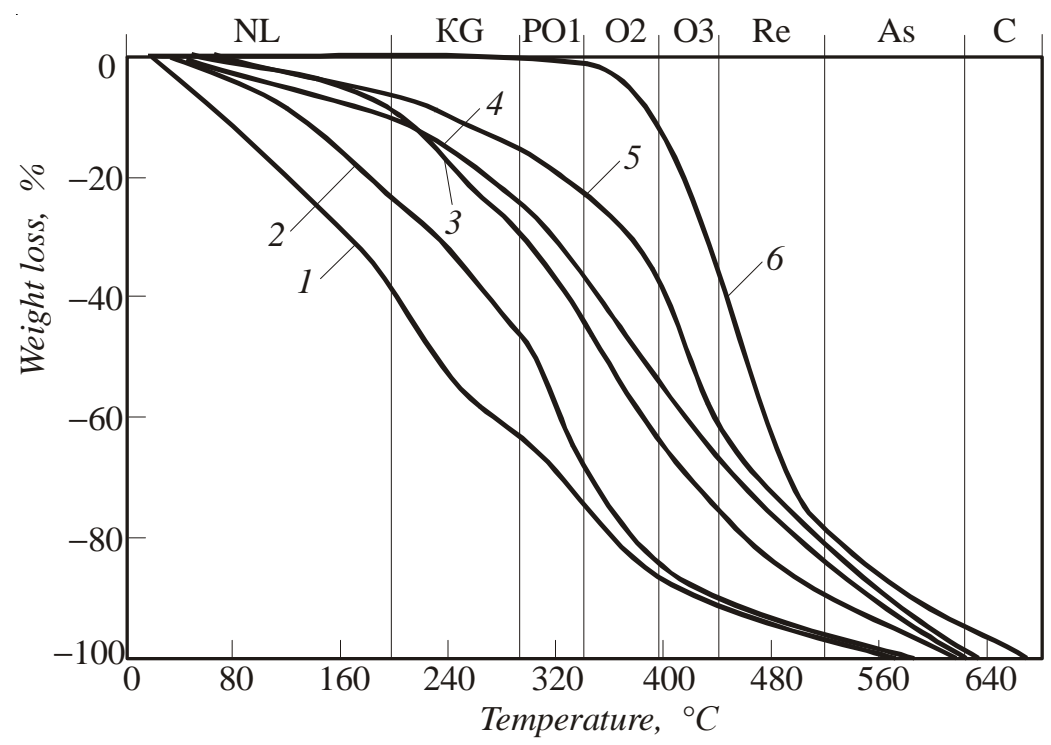

Fig. 2. Weight loss curves of crudes and products of their separation: 1, 2, 3, 4) Caspian, Vietnam, Tatar, and Snegirevsk crudes; 5) O-100 atmospheric resid; 6) vacuum resid; $N L, K G$ - naphtha-ligroin and kerosene-gasoil cuts; $P O 1$ - paraffins + light oil cuts; O2, O3 - middle and heavy oil cuts; $R e$ - resins; As - asphaltenes; $C$ - carbenes. 
the properties of a given component are based on the different vaporizability of the components [3]. The same principle is the basis of the TG-DSC method, designed for the same purposes but significantly differing in instrumentation and operating methods.

A special feature of modern calorimetric methods consists of the use of samples of very low weights - of the order of $5-20 \mathrm{mg} /$, which increases the quality of the thermograms recorded. The weight loss of such samples when the temperature changes is usually recorded with very expensive and not always readily available proprietary thermobalances.

A 13-15 mg sample heated at a given constant rate was weighed after the given temperature was attained to determine the weight loss on an ordinary analytical balance. The operation was repeated with another sample from the same lot, heating it to a higher temperature. The entire range of investigated temperatures with a step of $30-50^{\circ}$ was covered with this method. After this, a curve was plotted in "weight loss, wt. \%-standard temperature, ${ }^{\circ} \mathrm{C} "$ coordinates.

The DSC curve of the object investigated in coordinates of "DT-T2" coordinates was first recorded on a Termodat scanning calorimeter of our design, where $\mathrm{D} T=T 1-T 2 ; T 1, T 2$ are the temperatures of the sample and standard. The experimental conditions are: heating a $10-12 \mathrm{mg}$ sample in air at the rate of $40-45^{\circ} / \mathrm{min}$. The TG and DSC curves were plotted on the same graph. The nature of the thermal reactions in the sample, i.e., the nature of the components, was interpreted with the DSC curve, and the temperature ranges of their oxidation was found. The percentage content of the components in the mixture was found with the TG curve.

The TG-DSC curves of Kazakh crude from the Blinov field are shown in Fig. 1. The temperature range of the experiment can be divided into two segments. Loss of the light (distillate) part of the crude takes place in the first and loss of the heavy part occurs in the second segment. The flash point $T_{\mathrm{fl}}=298^{\circ} \mathrm{C}$ of the heavy residue was used as the boundary between the two segments.

In the example given, the sample lost $54 \%$ of the weight without marked thermal effects before $T_{\mathrm{fl}}$.

Further weight loss, beginning from $T_{\mathrm{fl}}$, was accompanied by a very pronounced exothermic effect: a peak with a steep initial front appeared on the DSC curve. Oxidation of the sample with heat release continued up to $586^{\circ} \mathrm{C}$. Three basic temperature regions can be distinguished on the DSC curve: $300-400^{\circ} \mathrm{C}-$ with a high heat of oxidation; $400-500^{\circ} \mathrm{C}$ - with a low heat of oxidation; $500-600^{\circ} \mathrm{C}$ - again with a high heat of oxidation.

The following interpretation of these events is possible.

As the temperature rose, first light naphtha and then heavier kerosene-gasoil cuts evaporated from the sample. Evaporation took place without important thermal effects: the uniform horizontal line on the DSC curve. Evaporation of these cuts was basically completed at $300^{\circ} \mathrm{C}$, and residue of the atmospheric type was found in the cup.

Oxidation of the residue began with ignition of the solid waxes in it, whose boiling point is above $280-300^{\circ} \mathrm{C}$, i.e., $\mathrm{C}_{18}-\mathrm{C}_{24}$ and higher $n$-paraffins. The following are subsequently successively oxidized: solid waxes + light oil cuts, paraffin-naphthenes, and naphthene-paraffins (middle and heavy oil cuts), mono-, bi-, and polycyclic aromatic compounds (light and middle resins), heavy resins, asphaltenes, carbenes.

The general data processing scheme is as follows. The graph with the curves was divided by vertical lines into a series of segments corresponding to the temperature ranges in which reactions of first evaporation and then oxidation of the sample cuts hypothetically take place. Evaporation ranges equal to IBP-140, 140-180, 180-240, and 240- $T_{\mathrm{b}}{ }^{\circ} \mathrm{C}$ are hypothesized for naphtha, ligroin, kerosene, and gasoil cuts.

The evaporation/oxidation temperatures of the components of the heavy resid were determined with the characteristic points on the DSC curve; they can be used to give the following assignments of the temperature ranges: 


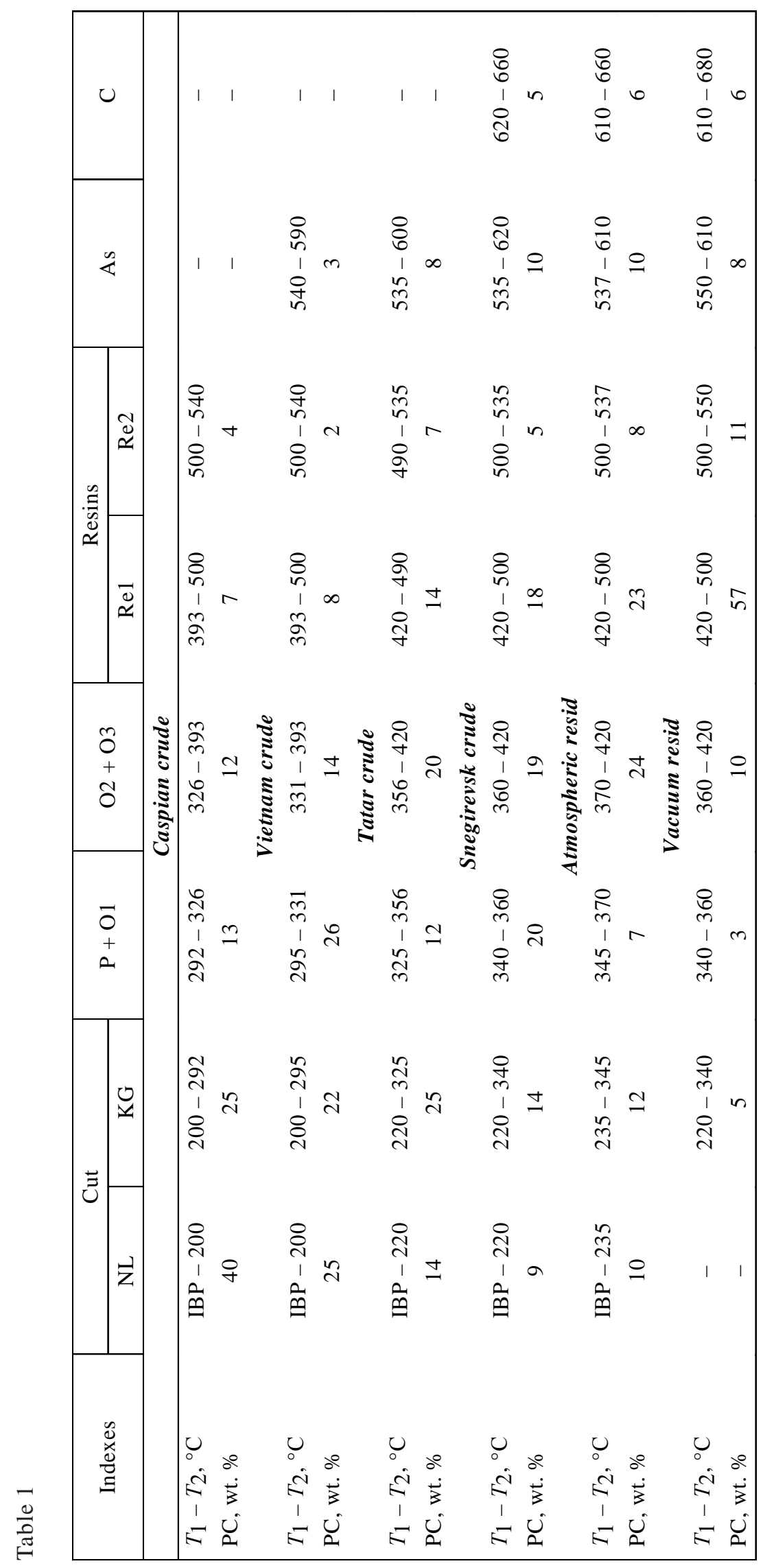


1) from the beginning to the apex of the peak - paraffins + light oil fraction $\mathrm{O} 1$;

2) from the apex of the peak to the point of inflection on the descending branch of the peak - middle oil cut $\mathrm{O} 2$;

3 ) between the point of inflection of the descending branch and the end of the peak - heavy oil cut O3;

4) between the end of the first peak and the beginning of the second - light and middle resins;

5) from the beginning of the second peak in the $500^{\circ} \mathrm{C}$ region to the point of inflection on the ascending branch - heavy resins (the "fur coat" of asphaltenes);

6) between the point of inflection on the ascending branch and the point of inflection on the descending branch of the peak - asphaltenes;

7) if present: between the point of inflection of the descending branch and the end of the peak - carbenes.

Since both paraffins and light oil cuts are oxidized in the first temperature interval, it is impossible to determine them separately with the TG-DSC curves. However, it was previously [1] shown that $n$-paraffins are oxidized on average with a high heat of $8000 \mathrm{~J} / \mathrm{g}$ while the hydrocarbons in the oils are oxidized with a high heat of $1800 \mathrm{~J} / \mathrm{g}$. If we assume that the experimental heat of oxidation $\Delta H_{\mathrm{ex}}$ on the segment of the ascending branch of the peak is additively made up of the heats of oxidation of $n$-paraffins $\Delta H_{\mathrm{p}}$ and oil hydrocarbons $\Delta H_{\mathrm{o}}$, then we can write the following system of equations with two unknowns - fractions $\alpha$ and $\beta$ of these components in the mixture:

$$
\left\{\begin{array}{l}
\boldsymbol{\Delta} \boldsymbol{H}_{\mathrm{ex}}=\boldsymbol{\alpha} \boldsymbol{\Delta} \boldsymbol{H}_{\mathrm{o}}+\boldsymbol{\beta} \boldsymbol{H}_{\mathrm{o}}=8000 \boldsymbol{\alpha}+1800 \boldsymbol{\beta} \\
\boldsymbol{\alpha}+\boldsymbol{\beta}=1
\end{array}\right.
$$

whose solution gives

$$
\begin{gathered}
\boldsymbol{\alpha}=\left(\boldsymbol{\Delta} \boldsymbol{H}_{\mathrm{ex}}-\boldsymbol{\Delta} \boldsymbol{H}_{\mathrm{o}}\right) /\left(\Delta \boldsymbol{H}_{\mathrm{p}}-\boldsymbol{\Delta} \boldsymbol{H}_{\mathrm{o}}\right)=\left(\boldsymbol{\Delta} \boldsymbol{H}_{\mathrm{ex}}-1800\right) / 6200 \\
\boldsymbol{\beta}=1-\boldsymbol{\alpha}
\end{gathered}
$$

After this, we can find the content of $n$-paraffins $n$-P and light oil cuts $\mathrm{O} 1$ in the PO cut with the equations:

$$
\begin{gathered}
n-P=\alpha P O \\
01=\boldsymbol{\beta P O}=(1-\alpha) P O
\end{gathered}
$$

The isoparaffin content in the $\mathrm{O} 2+\mathrm{O} 3$ oil cut (wax content of the oil) can also be determined with the same equations, but with other values of the specific heat: $\Delta H_{\mathrm{p}}=12,200 \mathrm{~J} / \mathrm{g}$ and $\Delta H_{\mathrm{o}}=2200 \mathrm{~J} / \mathrm{g}$. These values correspond to the average heats of oxidation of isoparaffins and heavy hydrocarbons in the oils. The fraction of isoparaffins in the oil cut is:

$$
\boldsymbol{\alpha}=\left(\Delta \boldsymbol{H}_{\mathrm{ex}}-2200\right) / 10,000
$$

The described TG-DSC method was used for determining the component composition of a series of crudes and their constituents; from light waxy crudes of the Caspian type to heavy residue of the vacuum resid type. Only the TG curves are shown in Fig. 2 to facilitate comparing the results of the study. 
Evaporation/oxidation intervals, ${ }^{\circ} \mathrm{C}$
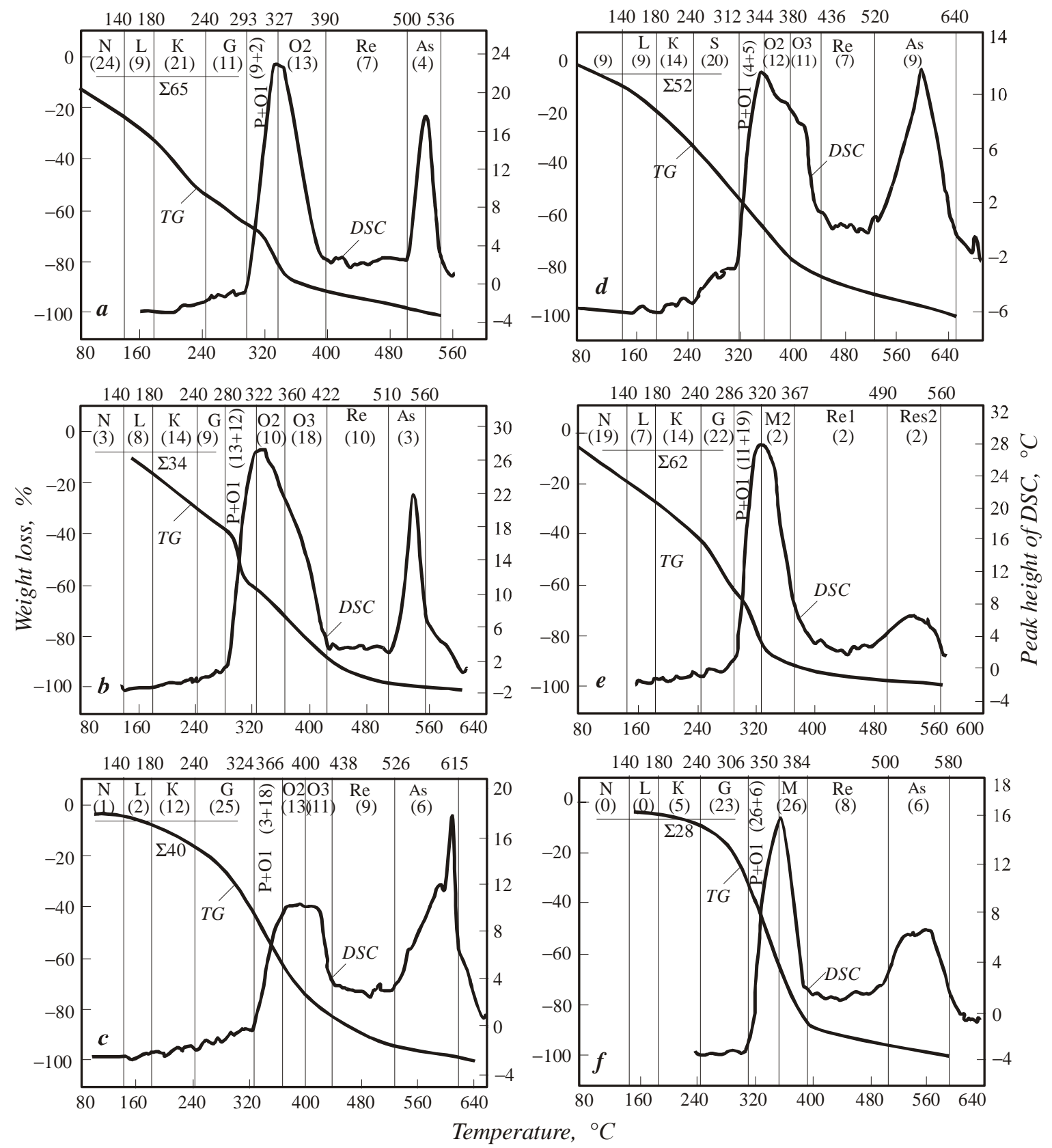

Fig. 3. TG-DSC curves of a series of crudes and products of their separation: a) Caspian crude (density at $\left.20^{\circ} \mathrm{C}, 826.6 \mathrm{~kg} / \mathrm{m}^{3}\right)$; b) crude from East Sarutayusk field $\left(862.7 \mathrm{~kg} / \mathrm{m}^{3}\right)$; c) crude from a Russian field $\left(981.2 \mathrm{~kg} / \mathrm{m}^{3}\right)$; d) West Siberian crude $\left(907.2 \mathrm{~kg} / \mathrm{m}^{3}\right)$; e) gas condensate $\left(768 \mathrm{~kg} / \mathrm{m}^{3}\right)$; f) vacuum distillate $\left(962.5 \mathrm{~kg} / \mathrm{m}^{3}\right)$; see Fig. 1 for other notation.

As the end point of the investigated object becomes higher, the curves are uniformly shifted to the right, from the region of the evaporation temperatures of the light cuts to the region of evaporation/oxidation of heavy cuts. The points of intersection of the TG curves with the lines which delimit the temperature regions of evaporation/ oxidation of the cuts move up. This indicates a decrease in the proportion of light cuts in the product and an increase in the proportion of heavy cuts. As a result, we obtain the highest resin and asphaltene content for the 
heaviest product, vacuum resid, and an important content of oil cuts (especially heavy) in the absence of distillate constituents and waxes.

The middle intervals of evaporation/oxidation of the components of the hydrocarbon mixtures are shown in Fig. 2. In reality, these intervals differ slightly for each product: the lighter the petroleum product, the lower the temperatures and the narrower the range of evaporation/oxidation of its cuts (see Table 1).

The refined values of the evaporation/oxidation intervals $T_{1}-T_{2}$ and potential content (PC) of the cuts are reported in Table 1. The data show a uniform decrease in the content of light distillates, an increase in the resin and asphaltene content, and the appearance of carbenes in going from light products to heavy products.

The evaporation temperatures of the kerosene-gasoil cut and the temperature intervals of evaporation/oxidation of waxes and oil cuts increase markedly. It is interesting to note that the curves of the different products never intersect, which indicates a common mechanism of the change in their component composition.

The crudes were qualitatively analyzed with the DSC curves (Fig. 3). A characteristic shape of the curve (oxidation profile) with which the crude can easily be identified (classified) corresponds to each class of crude oils. For example, paraffin-base crudes (mature crudes of important geological age) are characterized by a high, narrow paraffin peak in the $300-400^{\circ} \mathrm{C}$ region with $T_{\mathrm{fl}}=300 \pm 10^{\circ} \mathrm{C}$ and a low narrow $500-540^{\circ} \mathrm{C}$ resin-asphaltene peak.

The narrowness of the paraffin peak and absence of a developed right shoulder in its structure indicate a low content of oil cuts in the sample and the asphaltene peak of small area shifted to low temperatures indicates a high degree of decomposition of the asphaltene cut and the closeness of its properties to heavy resins.

On the other hand, a low left and broad right shoulder in the structure of the paraffin-oil peak, shift of the right shoulder to the high-temperature region (to $420-430^{\circ} \mathrm{C}$ when heavy oils are present in the sample), and a broad asphaltene peak of important area shifted to the $520-640^{\circ} \mathrm{C}$ region are characteristic of naphthene and naphthene-aromatic crudes.

These thermal properties indicate the young geological age of the crude in which decomposition of asphaltene and oil cuts and their transformation into paraffins and the light part are in the middle or initial stage of development.

We can draw the following conclusions based on the results of the studies.

Caspian crude contains a significant proportion of the light part with predominance of the naphtha cut, a lot of $n$-paraffins with $T_{\mathrm{fl}}=293^{\circ} \mathrm{C}$, few light oil cuts, much more middle, and absolutely no heavy cuts. The resin and asphaltene content constitutes $11 \%$. The asphaltene peak is small and shifted to the low-temperature region. The crude can be classified as light high-wax with a high degree of catagenetic transformation, i.e., of important geological age.

The crude from the East Sarutayusk field contains in addition to $n$-paraffins an important amount of light, middle, and heavy oil cuts. The distillate part is small. The asphaltene peak is small, and the resin-asphaltene (RA) content is $13 \%$. The crude is paraffin-naphthene base, low catagenetic transformation, and sapropelic genesis.

The crude from the Russian field contains $3 \%$ isoparaffins with $T_{\mathrm{fl}}=324^{\circ} \mathrm{C}$, $42 \%$ wide oil cut with a $324-438^{\circ} \mathrm{C}$ oxidation range, $40 \%$ light part, and $15 \% \mathrm{RA}$. The absence of $n$-paraffins indicates the humus genesis of the crude. Predominance of the oil cut and the average content of RA and light part allow assigning the crude to naphthene base of medium catagenetic transformation.

The West Siberian crude (blend) contains $4 \% n$ - or isoparaffins with $T_{\mathrm{fl}}=312^{\circ} \mathrm{C}$ and $16 \% \mathrm{RA}$. The crude is naphthene-paraffin base, with a medium degree of transformation, of mixed sapropelic-humus genesis. 
The gas condensate is a light crude of a high degree of transformation in which all of the heavy components have been converted into the light (paraffin and oil) and distillate part. It contains many $n$-paraffins (19\%) and light oil cuts (15\%) with a narrow oxidation range of $285-367^{\circ} \mathrm{C}$, very little $(4 \%)$ of middle and heavy resins, and a significant proportion (62\%) of the light part.

Vacuum distillate - the product of vacuum distillation of the last cuts of the crude basically contains middle oil cuts and high-boiling waxes.

\section{REFERENCES}

1. Yu. L. Shishkin, Thermochim. Acta, 441, No. 2, 162-167 (2006).

2. Yu. L. Shishkin, Ibid., 440, No. 2, 156-165 (2006).

3. V. A. Proskuryakov (ed.), Chemistry of Oil and Gas [in Russian], Khimiya, Leningrad (1989). 